Network Working Group

Request for Comments: 1944

Category: Informational
S. Bradner

Harvard University

J. McQuaid

Bay Networks

May 1996

Benchmarking Methodology for Network Interconnect Devices

Status of This Memo

This memo provides information for the Internet community. This memo does not specify an Internet standard of any kind. Distribution of this memo is unlimited.

Abstract

This document discusses and defines a number of tests that may be used to describe the performance characteristics of a network interconnecting device. In addition to defining the tests this document also describes specific formats for reporting the results of the tests. Appendix A lists the tests and conditions that we believe should be included for specific cases and gives additional information about testing practices. Appendix B is a reference listing of maximum frame rates to be used with specific frame sizes on various media and Appendix C gives some examples of frame formats to be used in testing.

1. Introduction

Vendors often engage in "specsmanship" in an attempt to give their products a better position in the marketplace. This often involves "smoke \& mirrors" to confuse the potential users of the products.

This document defines a specific set of tests that vendors can use to measure and report the performance characteristics of network devices. The results of these tests will provide the user comparable data from different vendors with which to evaluate these devices.

A previous document, "Benchmarking Terminology for Network Interconnect Devices" (RFC 1242), defined many of the terms that are used in this document. The terminology document should be consulted before attempting to make use of this document. 


\section{Real world}

In producing this document the authors attempted to keep in mind the requirement that apparatus to perform the described tests must actually be built. We do not know of "off the shelf" equipment available to implement all of the tests but it is our opinion that such equipment can be constructed.

3. Tests to be run

There are a number of tests described in this document. Not all of the tests apply to all types of devices under test (DUTs). Vendors should perform all of the tests that can be supported by a specific type of product. The authors understand that it will take a considerable period of time to perform all of the recommended tests nder all of the recommended conditions. We believe that the results are worth the effort. Appendix A lists some of the tests and conditions that we believe should be included for specific cases.

4. Evaluating the results

Performing all of the recommended tests will result in a great deal of data. Much of this data will not apply to the evaluation of the devices under each circumstance. For example, the rate at which a router forwards IPX frames will be of little use in selecting a router for an environment that does not (and will not) support that protocol. Evaluating even that data which is relevant to a particular network installation will require experience which may not be readily available. Furthermore, selection of the tests to be run and evaluation of the test data must be done with an understanding of generally accepted testing practices regarding repeatability, variance and statistical significance of small numbers of trials.

5. Requirements

In this document, the words that are used to define the significance of each particular requirement are capitalized. These words are:

* "MUST" This word, or the words "REQUIRED" and "SHALL" mean that the item is an absolute requirement of the specification.

* "SHOULD" This word or the adjective "RECOMMENDED" means that there may exist valid reasons in particular circumstances to ignore this item, but the full implications should be understood and the case carefully weighed before choosing a different course.

* "MAY" This word or the adjective "OPTIONAL" means that this item is truly optional. One vendor may choose to include the item because 
a particular marketplace requires it or because it enhances the product, for example; another vendor may omit the same item.

An implementation is not compliant if it fails to satisfy one or more of the MUST requirements for the protocols it implements. An implementation that satisfies all the MUST and all the SHOULD requirements for its protocols is said to be "unconditionally compliant"; one that satisfies all the MUST requirements but not all the SHOULD requirements for its protocols is said to be

"conditionally compliant".

6. Test set up

The ideal way to implement this series of tests is to use a tester with both transmitting and receiving ports. Connections are made from the sending ports of the tester to the receiving ports of the DUT and from the sending ports of the DUT back to the tester. (see Figure 1) Since the tester both sends the test traffic and receives it back, after the traffic has been forwarded but the DUT, the tester can easily determine if all of the transmitted packets were received and verify that the correct packets were received. The same functionality can be obtained with separate transmitting and receiving devices (see Figure 2) but unless they are remotely controlled by some computer in a way that simulates the single tester, the labor required to accurately perform some of the tests (particularly the throughput test) can be prohibitive.
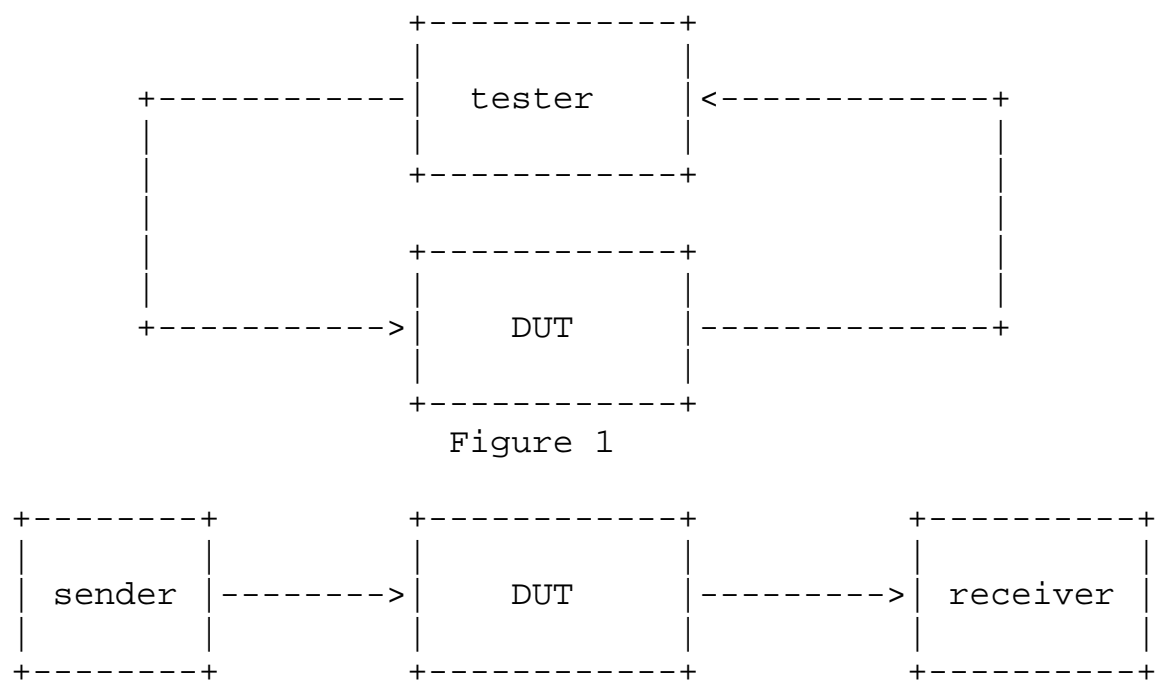

Figure 2 
6.1 Test set up for multiple media types

Two different setups could be used to test a DUT which is used in real-world networks to connect networks of differing media type, local Ethernet to a backbone FDDI ring for example. The tester could support both media types in which case the set up shown in Figure 1 would be used.

Two identical DUTs are used in the other test set up. (see Figure 3) In many cases this set up may more accurately simulate the real world. For example, connecting two LANs together with a WAN link or high speed backbone. This set up would not be as good at simulating a system where clients on a Ethernet LAN were interacting with a server on an FDDI backbone.

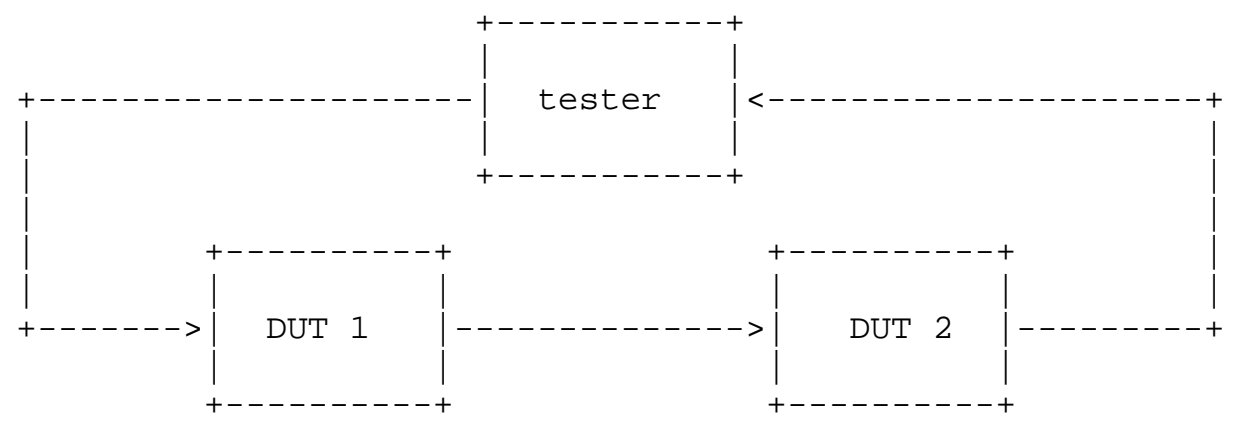

Figure 3

7. DUT set up

Before starting to perform the tests, the DUT to be tested MUST be configured following the instructions provided to the user. Specifically, it is expected that all of the supported protocols will be configured and enabled during this set up (See Appendix A). It is expected that all of the tests will be run without changing the configuration or setup of the DUT in any way other than that required to do the specific test. For example, it is not acceptable to change the size of frame handling buffers between tests of frame handling rates or to disable all but one transport protocol when testing the throughput of that protocol. It is necessary to modify the configuration when starting a test to determine the effect of filters on throughput, but the only change MUST be to enable the specific filter. The DUT set up SHOULD include the normally recommended routing update intervals and keep alive frequency. The specific version of the software and the exact DUT configuration, including what functions are disabled, used during the tests MUST be included as part of the report of the results. 
8. Frame formats

The formats of the test frames to use for TCP/IP over Ethernet are shown in Appendix C: Test Frame Formats. These exact frame formats SHOULD be used in the tests described in this document for this protocol/media combination and that these frames will be used as a template for testing other protocol/media combinations. The specific formats that are used to define the test frames for a particular test series MUST be included in the report of the results.

9. Frame sizes

All of the described tests SHOULD be performed at a number of frame sizes. Specifically, the sizes SHOULD include the maximum and minimum legitimate sizes for the protocol under test on the media under test and enough sizes in between to be able to get a full characterization of the DUT performance. Except where noted, at least five frame sizes SHOULD be tested for each test condition.

Theoretically the minimum size UDP Echo request frame would consist of an IP header (minimum length 20 octets), a UDP header (8 octets) and whatever MAC level header is required by the media in use. The theoretical maximum frame size is determined by the size of the length field in the IP header. In almost all cases the actual maximum and minimum sizes are determined by the limitations of the media.

In theory it would be ideal to distribute the frame sizes in a way that would evenly distribute the theoretical frame rates. These recommendations incorporate this theory but specify frame sizes which are easy to understand and remember. In addition, many of the same frame sizes are specified on each of the media types to allow for easy performance comparisons.

Note: The inclusion of an unrealistically small frame size on some of the media types (i.e. with little or no space for data) is to help characterize the per-frame processing overhead of the DUT.

9.1 Frame sizes to be used on Ethernet

$64,128,256,512,1024,1280,1518$

These sizes include the maximum and minimum frame sizes permitted by the Ethernet standard and a selection of sizes between these extremes with a finer granularity for the smaller frame sizes and higher frame rates. 
9.2 Frame sizes to be used on $4 \mathrm{Mb}$ and $16 \mathrm{Mb}$ token ring

$54,64,128,256,1024,1518,2048,4472$

The frame size recommendations for token ring assume that there is no RIF field in the frames of routed protocols. A RIF field would be present in any direct source route bridge performance test. The minimum size frame for UDP on token ring is 54 octets. The maximum size of 4472 octets is recommended for $16 \mathrm{Mb}$ token ring instead of the theoretical size of $17.9 \mathrm{~Kb}$ because of the size limitations imposed by many token ring interfaces. The reminder of the sizes are selected to permit direct comparisons with other types of media. An IP (i.e. not UDP) frame may be used in addition if a higher data rate is desired, in which case the minimum frame size is 46 octets.

9.3 Frame sizes to be used on FDDI

$54,64,128,256,1024,1518,2048,4472$

The minimum size frame for UDP on FDDI is 53 octets, the minimum size of 54 is recommended to allow direct comparison to token ring performance. The maximum size of 4472 is recommended instead of the theoretical maximum size of 4500 octets to permit the same type of comparison. An IP (i.e. not UDP) frame may be used in addition if a higher data rate is desired, in which case the minimum frame size is 45 octets.

\subsection{Frame sizes in the presence of disparate MTUs}

When the interconnect DUT supports connecting links with disparate MTUs, the frame sizes for the link with the *larger* MTU SHOULD be used, up to the limit of the protocol being tested. If the interconnect DUT does not support the fragmenting of frames in the presence of MTU mismatch, the forwarding rate for that frame size shall be reported as zero.

For example, the test of IP forwarding with a bridge or router that joins FDDI and Ethernet should use the frame sizes of FDDI when going from the FDDI to the Ethernet link. If the bridge does not support IP fragmentation, the forwarding rate for those frames too large for Ethernet should be reported as zero.

10. Verifying received frames

The test equipment SHOULD discard any frames received during a test run that are not actual forwarded test frames. For example, keepalive and routing update frames SHOULD NOT be included in the count 
of received frames. In any case, the test equipment sHoulD verify the length of the received frames and check that they match the expected length.

Preferably, the test equipment SHOULD include sequence numbers in the transmitted frames and check for these numbers on the received frames. If this is done, the reported results sHould include in addition to the number of frames dropped, the number of frames that were received out of order, the number of duplicate frames received and the number of gaps in the received frame numbering sequence. This functionality is required for some of the described tests.

\section{Modifiers}

It might be useful to know the DUT performance under a number of conditions; some of these conditions are noted below. The reported results SHOULD include as many of these conditions as the test equipment is able to generate. The suite of tests sHould be first run without any modifying conditions and then repeated under each of the conditions separately. To preserve the ability to compare the results of these tests any frames that are required to generate the modifying conditions (management queries for example) will be included in the same data stream as the normal test frames in place of one of the test frames and not be supplied to the DUT on a separate network port.

\subsection{Broadcast frames}

In most router designs special processing is required when frames addressed to the hardware broadcast address are received. In bridges (or in bridge mode on routers) these broadcast frames must be flooded to a number of ports. The stream of test frames SHOULD be augmented with 1\% frames addressed to the hardware broadcast address. The frames sent to the broadcast address should be of a type that the router will not need to process. The aim of this test is to determine if there is any effect on the forwarding rate of the other data in the stream. The specific frames that should be used are included in the test frame format document. The broadcast frames SHOULD be evenly distributed throughout the data stream, for example, every 100th frame.

The same test SHOULD be performed on bridge-like DUTs but in this case the broadcast packets will be processed and flooded to all outputs.

It is understood that a level of broadcast frames of 1\% is much higher than many networks experience but, as in drug toxicity evaluations, the higher level is required to be able to gage the 
effect which would otherwise often fall within the normal variability of the system performance. Due to design factors some test equipment will not be able to generate a level of alternate frames this low. In these cases the percentage SHOULD be as small as the equipment can provide and that the actual level be described in the report of the test results.

\subsection{Management frames}

Most data networks now make use of management protocols such as SNMP. In many environments there can be a number of management stations sending queries to the same DUT at the same time.

The stream of test frames SHOULD be augmented with one management query as the first frame sent each second during the duration of the trial. The result of the query must fit into one response frame. The response frame SHOULD be verified by the test equipment. One example of the specific query frame that should be used is shown in Appendix $C$.

\subsection{Routing update frames}

The processing of dynamic routing protocol updates could have a significant impact on the ability of a router to forward data frames. The stream of test frames SHOULD be augmented with one routing update frame transmitted as the first frame transmitted during the trial. Routing update frames SHOULD be sent at the rate specified in Appendix C for the specific routing protocol being used in the test. Two routing update frames are defined in Appendix C for the TCP/IP over Ethernet example. The routing frames are designed to change the routing to a number of networks that are not involved in the forwarding of the test data. The first frame sets the routing table state to "A", the second one changes the state to "B". The frames MUST be alternated during the trial.

The test SHOULD verify that the routing update was processed by the DUT.

\subsection{Filters}

Filters are added to routers and bridges to selectively inhibit the forwarding of frames that would normally be forwarded. This is usually done to implement security controls on the data that is accepted between one area and another. Different products have different capabilities to implement filters. 
The DUT SHOULD be first configured to add one filter condition and the tests performed. This filter SHOULD permit the forwarding of the test data stream. In routers this filter sHould be of the form:

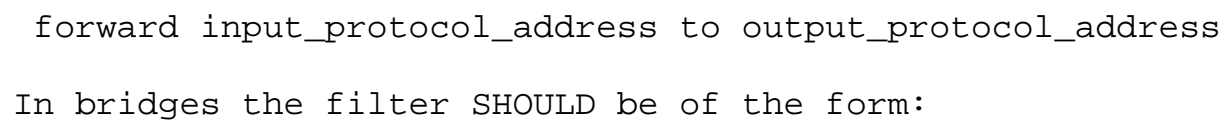

\subsubsection{Filter Addresses}

Two sets of filter addresses are required, one for the single filter case and one for the 25 filter case.

The single filter case should permit traffic from IP address 198.18.1.2 to IP address 198.19.65.2 and deny all other traffic.

The 25 filter case should follow the following sequence.

deny aa.ba.1.1 to aa.ba.100.1

deny aa.ba.2.2 to aa.ba.101.2

deny aa.ba.3.3 to aa.ba.103.3

$$
\text { ... }
$$

deny aa.ba.12.12 to aa.ba.112.12

allow aa.bc.1.2 to aa.bc.65.1

deny aa.ba.13.13 to aa.ba.113.13

deny aa.ba.14.14 to aa.ba.114.14 


\author{
deny aa.ba.24.24 to aa.ba.124.24 \\ deny all else
}

$\cdots$

All previous filter conditions should be cleared from the router before this sequence is entered. The sequence is selected to test to see if the router sorts the filter conditions or accepts them in the order that they were entered. Both of these procedures will result in a greater impact on performance than will some form of hash coding.

12. Protocol addresses

It is easier to implement these tests using a single logical stream of data, with one source protocol address and one destination protocol address, and for some conditions like the filters described above, a practical requirement. Networks in the real world are not limited to single streams of data. The test suite SHOULD be first run with a single protocol (or hardware for bridge tests) source and destination address pair. The tests SHOULD then be repeated with using a random destination address. While testing routers the addresses SHOULD be random and uniformly distributed over a range of 256 networks and random and uniformly distributed over the full MAC range for bridges. The specific address ranges to use for IP are shown in Appendix C.

13. Route set Up

It is not reasonable that all of the routing information necessary to forward the test stream, especially in the multiple address case, will be manually set up. At the start of each trial a routing update MUST be sent to the DUT. This routing update MUST include all of the network addresses that will be required for the trial. All of the addresses SHOULD resolve to the same "next-hop". Normally this will be the address of the receiving side of the test equipment. This routing update will have to be repeated at the interval required by the routing protocol being used. An example of the format and repetition interval of the update frames is given in Appendix $\mathrm{C}$.

14. Bidirectional traffic

Normal network activity is not all in a single direction. To test the bidirectional performance of a DUT, the test series SHOULD be run with the same data rate being offered from each direction. The sum of the data rates should not exceed the theoretical limit for the media. 
15. Single stream path

The full suite of tests SHOULD be run along with whatever modifier conditions that are relevant using a single input and output network port on the DUT. If the internal design of the DUT has multiple distinct pathways, for example, multiple interface cards each with multiple network ports, then all possible types of pathways SHouLD be tested separately.

16. Multi-port

Many current router and bridge products provide many network ports in the same module. In performing these tests first half of the ports are designated as "input ports" and half are designated as "output ports". These ports SHOULD be evenly distributed across the DUT architecture. For example if a DUT has two interface cards each of which has four ports, two ports on each interface card are designated as input and two are designated as output. The specified tests are run using the same data rate being offered to each of the input ports. The addresses in the input data streams SHOULD be set so that a frame will be directed to each of the output ports in sequence so that all "output" ports will get an even distribution of packets from this input. The same configuration MAY be used to perform a bidirectional multi-stream test. In this case all of the ports are considered both input and output ports and each data stream MUST consist of frames addressed to all of the other ports.

Consider the following 6 port DUT:

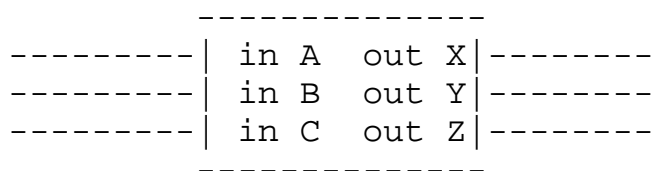

The addressing of the data streams for each of the inputs SHOULD be:

stream sent to input $A$ : packet to out $X$, packet to out $Y$, packet to out $Z$ stream sent to input $B$ : packet to out $X$, packet to out $Y$, packet to out $Z$ stream sent to input $C$ packet to out $\mathrm{X}$, packet to out $\mathrm{Y}$, packet to out $\mathrm{Z}$

Note that these streams each follow the same sequence so that 3 packets will arrive at output $X$ at the same time, then 3 packets at $Y$, then 3 packets at $Z$. This procedure ensures that, as in the real world, the DUT will have to deal with multiple packets addressed to 
the same output at the same time.

17. Multiple protocols

This document does not address the issue of testing the effects of a mixed protocol environment other than to suggest that if such tests are wanted then frames SHOULD be distributed between all of the test protocols. The distribution MAY approximate the conditions on the network in which the DUT would be used.

18. Multiple frame sizes

This document does not address the issue of testing the effects of a mixed frame size environment other than to suggest that if such tests are wanted then frames SHOULD be distributed between all of the listed sizes for the protocol under test. The distribution MAY approximate the conditions on the network in which the DUT would be used. The authors do not have any idea how the results of such a test would be interpreted other than to directly compare multiple DUTs in some very specific simulated network.

19. Testing performance beyond a single DUT.

In the performance testing of a single DUT, the paradigm can be described as applying some input to a DUT and monitoring the output. The results of which can be used to form a basis of characterization of that device under those test conditions.

This model is useful when the test input and output are homogenous (e.g., 64-byte IP, 802.3 frames into the DUT; 64 byte IP, 802.3 frames out), or the method of test can distinguish between dissimilar input/output. (E.g., 1518 byte IP, 802.3 frames in; 576 byte, fragmented IP, X.25 frames out.)

By extending the single DUT test model, reasonable benchmarks regarding multiple DUTs or heterogeneous environments may be collected. In this extension, the single DUT is replaced by a system of interconnected network DUTs. This test methodology would support the benchmarking of a variety of device/media/service/protocol combinations. For example, a configuration for a LAN-to-WAN-to-LAN test might be:

(1) $802.3->$ DUT $1 \rightarrow$ X.25 @ $64 \mathrm{kbps} \rightarrow$ DUT $2 \rightarrow 802.3$

Or a mixed LAN configuration might be:

(2) $802.3 \rightarrow$ DUT $1 \rightarrow$ FDDI $\rightarrow$ DUT $2 \rightarrow$ FDDI $\rightarrow$ DUT $3 \rightarrow 802.3$ 
In both examples 1 and 2, end-to-end benchmarks of each system could be empirically ascertained. Other behavior may be characterized through the use of intermediate devices. In example 2, the configuration may be used to give an indication of the FDDI to FDDI capability exhibited by DUT 2 .

Because multiple DUTs are treated as a single system, there are limitations to this methodology. For instance, this methodology may yield an aggregate benchmark for a tested system. That benchmark alone, however, may not necessarily reflect asymmetries in behavior between the DUTs, latencies introduce by other apparatus (e.g., CSUs/DSUs, switches), etc.

Further, care must be used when comparing benchmarks of different systems by ensuring that the DUTs' features/configuration of the tested systems have the appropriate common denominators to allow comparison.

20. Maximum frame rate

The maximum frame rates that should be used when testing LAN connections SHOULD be the listed theoretical maximum rate for the frame size on the media.

The maximum frame rate that should be used when testing WAN connections SHOULD be greater than the listed theoretical maximum rate for the frame size on that speed connection. The higher rate for WAN tests is to compensate for the fact that some vendors employ various forms of header compression.

A list of maximum frame rates for LAN connections is included in Appendix B.

\section{Bursty traffic}

It is convenient to measure the DUT performance under steady state load but this is an unrealistic way to gauge the functioning of a DUT since actual network traffic normally consists of bursts of frames. Some of the tests described below SHOULD be performed with both steady state traffic and with traffic consisting of repeated bursts of frames. The frames within a burst are transmitted with the minimum legitimate inter-frame gap.

The objective of the test is to determine the minimum interval between bursts which the DUT can process with no frame loss. During each test the number of frames in each burst is held constant and the inter-burst interval varied. Tests SHOULD be run with burst sizes of $16,64,256$ and 1024 frames. 
22. Frames per token

Although it is possible to configure some token ring and FDDI interfaces to transmit more than one frame each time that the token is received, most of the network devices currently available transmit only one frame per token. These tests SHOULD first be performed while transmitting only one frame per token.

Some current high-performance workstation servers do transmit more than one frame per token on FDDI to maximize throughput. Since this may be a common feature in future workstations and servers, interconnect devices with FDDI interfaces SHOULD be tested with 1, 4, 8, and 16 frames per token. The reported frame rate SHOULD be the average rate of frame transmission over the total trial period.

\section{Trial description}

A particular test consists of multiple trials. Each trial returns one piece of information, for example the loss rate at a particular input frame rate. Each trial consists of a number of phases:

a) If the DUT is a router, send the routing update to the "input" port and pause two seconds to be sure that the routing has settled.

b) Send the "learning frames" to the "output" port and wait 2 seconds to be sure that the learning has settled. Bridge learning frames are frames with source addresses that are the same as the destination addresses used by the test frames. Learning frames for other protocols are used to prime the address resolution tables in the DUT. The formats of the learning frame that should be used are shown in the Test Frame Formats document.

c) Run the test trial.

d) Wait for two seconds for any residual frames to be received.

e) Wait for at least five seconds for the DUT to restabilize.

24. Trial duration

The aim of these tests is to determine the rate continuously supportable by the DUT. The actual duration of the test trials must be a compromise between this aim and the duration of the benchmarking test suite. The duration of the test portion of each trial SHouLD be at least 60 seconds. The tests that involve some form of "binary search", for example the throughput test, to determine the exact result MAY use a shorter trial duration to minimize the length of the search procedure, but the final determination SHOULD be made with 
full length trials.

25. Address resolution

The DUT SHOULD be able to respond to address resolution requests sent by the DUT wherever the protocol requires such a process.

26. Benchmarking tests:

Note: The notation "type of data stream" refers to the above
modifications to a frame stream with a constant inter-frame gap, for
example, the addition of traffic filters to the configuration of the
DUT.

26.1 Throughput

Objective:

To determine the DUT throughput as defined in RFC 1242.

Procedure:

Send a specific number of frames at a specific rate through the DUT and then count the frames that are transmitted by the DUT. If the count of offered frames is equal to the count of received frames, the rate of the offered stream is raised and the test rerun. If fewer frames are received than were transmitted, the rate of the offered stream is reduced and the test is rerun.

The throughput is the fastest rate at which the count of test frames transmitted by the DUT is equal to the number of test frames sent to it by the test equipment.

Reporting format:

The results of the throughput test SHOULD be reported in the form of a graph. If it is, the $x$ coordinate SHOULD be the frame size, the $y$ coordinate SHOULD be the frame rate. There SHOULD be at least two lines on the graph. There SHOULD be one line showing the theoretical frame rate for the media at the various frame sizes. The second line SHOULD be the plot of the test results. Additional lines MAY be used on the graph to report the results for each type of data stream tested. Text accompanying the graph SHOULD indicate the protocol, data stream format, and type of media used in the tests.

We assume that if a single value is desired for advertising purposes the vendor will select the rate for the minimum frame size for the media. If this is done then the figure MUST be expressed in frames per second. The rate MAY also be expressed in bits (or bytes) per second if the vendor so desires. The 
statement of performance MUST include a/ the measured maximum frame rate, b/ the size of the frame used, c/ the theoretical limit of the media for that frame size, and $d /$ the type of protocol used in the test. Even if a single value is used as part of the advertising copy, the full table of results sHoulD be included in the product data sheet.

\subsection{Latency}

Objective:

To determine the latency as defined in RFC 1242 .

Procedure:

First determine the throughput for DUT at each of the listed frame sizes. Send a stream of frames at a particular frame size through the DUT at the determined throughput rate to a specific destination. The stream SHOULD be at least 120 seconds in duration. An identifying tag SHOULD be included in one frame after 60 seconds with the type of tag being implementation dependent. The time at which this frame is fully transmitted is recorded (timestamp A). The receiver logic in the test equipment MUST recognize the tag information in the frame stream and record the time at which the tagged frame was received (timestamp B).

The latency is timestamp B minus timestamp A as per the relevant definition frm RFC 1242, namely latency as defined for store and forward devices or latency as defined for bit forwarding devices.

The test MUST be repeated at least 20 times with the reported value being the average of the recorded values.

This test SHOULD be performed with the test frame addressed to the same destination as the rest of the data stream and also with each of the test frames addressed to a new destination network.

Reporting format:

The report MUST state which definition of latency (from RFC 1242) was used for this test. The latency results SHOULD be reported in the format of a table with a row for each of the tested frame sizes. There SHOULD be columns for the frame size, the rate at which the latency test was run for that frame size, for the media types tested, and for the resultant latency values for each type of data stream tested. 


\subsection{Frame loss rate}

Objective:

To determine the frame loss rate, as defined in RFC 1242, of a DUT throughout the entire range of input data rates and frame sizes.

Procedure:

Send a specific number of frames at a specific rate through the DUT to be tested and count the frames that are transmitted by the DUT. The frame loss rate at each point is calculated using the following equation:

( ( input_count - output_count ) *100 ) / input_count

The first trial SHOULD be run for the frame rate that corresponds to $100 \%$ of the maximum rate for the frame size on the input media. Repeat the procedure for the rate that corresponds to $90 \%$ of the maximum rate used and then for $80 \%$ of this rate. This sequence SHOULD be continued (at reducing 10\% intervals) until there are two successive trials in which no frames are lost. The maximum granularity of the trials MUST be $10 \%$ of the maximum rate, a finer granularity is encouraged.

Reporting format:

The results of the frame loss rate test SHOULD be plotted as a graph. If this is done then the $\mathrm{X}$ axis MUST be the input frame rate as a percent of the theoretical rate for the media at the specific frame size. The $Y$ axis MUST be the percent loss at the particular input rate. The left end of the $\mathrm{X}$ axis and the bottom of the $Y$ axis MUST be 0 percent; the right end of the $X$ axis and the top of the $Y$ axis MUST be 100 percent. Multiple lines on the graph MAY used to report the frame loss rate for different frame sizes, protocols, and types of data streams.

Note: See section 18 for the maximum frame rates that SHouLD be used.

\subsection{Back-to-back frames}

Objective:

To characterize the ability of a DUT to process back-to-back frames as defined in RFC 1242 .

Procedure:

Send a burst of frames with minimum inter-frame gaps to the DUT and count the number of frames forwarded by the DUT. If the count of transmitted frames is equal to the number of frames forwarded the length of the burst is increased and the test is rerun. If 
the number of forwarded frames is less than the number transmitted, the length of the burst is reduced and the test is rerun.

The back-to-back value is the number of frames in the longest burst that the DUT will handle without the loss of any frames. The trial length MUST be at least 2 seconds and SHOULD be repeated at least 50 times with the average of the recorded values being reported.

Reporting format:

The back-to-back results SHOULD be reported in the format of a table with a row for each of the tested frame sizes. There SHOULD be columns for the frame size and for the resultant average frame count for each type of data stream tested. The standard deviation for each measurement MAY also be reported.

\subsection{System recovery}

Objective:

To characterize the speed at which a DUT recovers from an overload condition.

Procedure:

First determine the throughput for a DUT at each of the listed

frame sizes.

Send a stream of frames at a rate 110\% of the recorded throughput rate or the maximum rate for the media, whichever is lower, for at least 60 seconds. At Timestamp A reduce the frame rate to 50\% of the above rate and record the time of the last frame lost (Timestamp B). The system recovery time is determined by subtracting Timestamp B from Timestamp A. The test SHOULD be repeated a number of times and the average of the recorded values being reported.

Reporting format: The system recovery results SHOULD be reported in the format of a table with a row for each of the tested frame sizes. There SHOULD be columns for the frame size, the frame rate used as the throughput rate for each type of data stream tested, and for the measured recovery time for each type of data stream tested.

\subsection{Reset}

Objective:

To characterize the speed at which a DUT recovers from a device or software reset. 


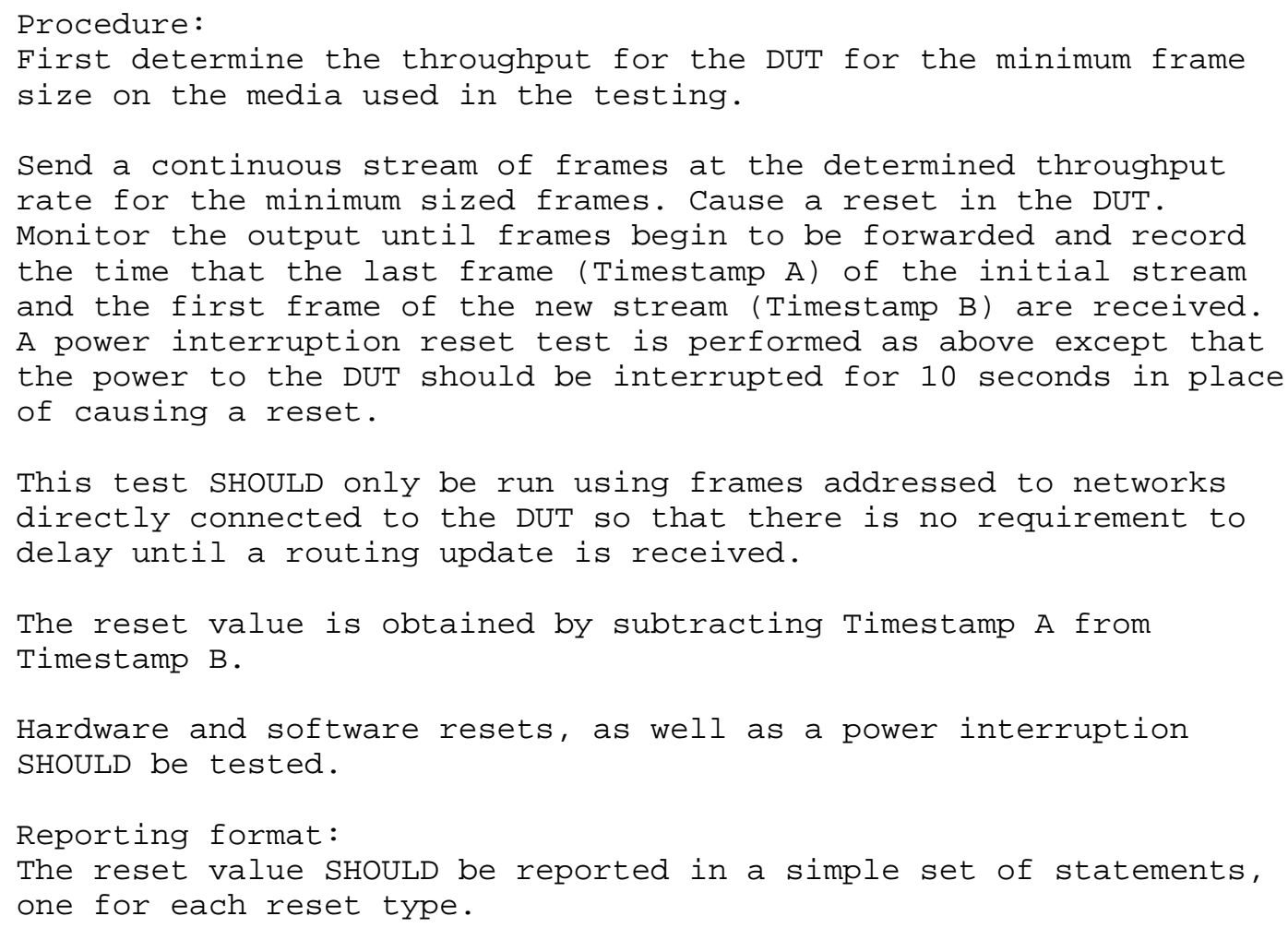

27. Security Considerations

Security issues are not addressed in this document. 
28. Editors' Addresses

Scott Bradner

Harvard University

1350 Mass. Ave, room 813

Cambridge, MA 02138

Phone +1 617 495-3864

Fax +1 617 496-8500

EMail: sob@harvard.edu

Jim McQuaid

Bay Networks

3 Federal Street

Billerica, MA 01821

Phone +1 508 436-3915

Fax: +1 508 670-8145

EMail: jmcquaid@baynetworks.com 


\section{Appendix A: Testing Considerations}

\section{A.1 Scope Of This Appendix}

This appendix discusses certain issues in the benchmarking methodology where experience or judgment may play a role in the tests selected to be run or in the approach to constructing the test with a particular DUT. As such, this appendix MUST not be read as an amendment to the methodology described in the body of this document but as a guide to testing practice.

1. Typical testing practice has been to enable all protocols to be tested and conduct all testing with no further configuration of protocols, even though a given set of trials may exercise only one protocol at a time. This minimizes the opportunities to "tune" a DUT for a single protocol.

2. The least common denominator of the available filter functions should be used to ensure that there is a basis for comparison between vendors. Because of product differences, those conducting and evaluating tests must make a judgment about this issue.

3. Architectural considerations may need to be considered. For example, first perform the tests with the stream going between ports on the same interface card and the repeat the tests with the stream going into a port on one interface card and out of a port on a second interface card. There will almost always be a best case and worst case configuration for a given DUT architecture.

4. Testing done using traffic streams consisting of mixed protocols has not shown much difference between testing with individual protocols. That is, if protocol A testing and protocol B testing give two different performance results, mixed protocol testing appears to give a result which is the average of the two.

5. Wide Area Network (WAN) performance may be tested by setting up two identical devices connected by the appropriate short- haul versions of the WAN modems. Performance is then measured between a LAN interface on one DUT to a LAN interface on the other DUT.

The maximum frame rate to be used for LAN-WAN-LAN configurations is a judgment that can be based on known characteristics of the overall system including compression effects, fragmentation, and gross link speeds. Practice suggests that the rate should be at least $110 \%$ of the slowest link speed. Substantive issues of testing compression itself are beyond the scope of this document. 
Appendix B: Maximum frame rates reference

$\begin{array}{lccr}\begin{array}{c}\text { (Provided by Roger Beeman, Cisco Systems) } \\ \text { Size } \\ \text { (bytes) }\end{array} & \begin{array}{c}\text { Ethernet } \\ \text { (pps) }\end{array} & \text { 16Mb Token Ring } & \begin{array}{r}\text { FDDI } \\ \text { (pps) }\end{array} \\ 64 & 14880 & 24691 & 152439 \\ 128 & 8445 & 13793 & 85616 \\ 256 & 4528 & 7326 & 45620 \\ 512 & 2349 & 3780 & 23585 \\ 768 & 1586 & 2547 & 15903 \\ 1024 & 1197 & 1921 & 11996 \\ 1280 & 961 & 1542 & 9630 \\ 1518 & 812 & 1302 & 8138\end{array}$

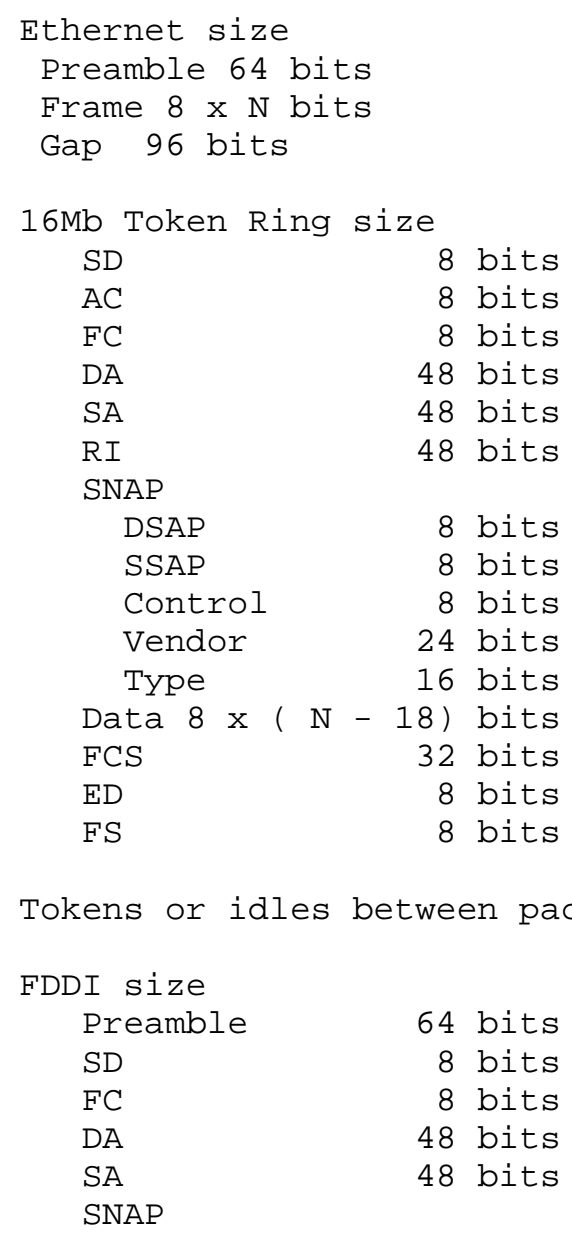




$\begin{array}{lr}\text { DSAP } & 8 \text { bits } \\ \text { SSAP } & 8 \text { bits } \\ \text { Control } & 8 \text { bits } \\ \text { Vendor } & 24 \text { bits } \\ \text { Type } & 16 \text { bits } \\ \text { Data } 8 \times(N-18) \text { bits } \\ \text { FCS } & 32 \text { bits } \\ \text { ED } & 4 \text { bits } \\ \text { FS } & 12 \text { bits }\end{array}$

Appendix C: Test Frame Formats

This appendix defines the frame formats that may be used with these tests. It also includes protocol specific parameters for TCP/IP over Ethernet to be used with the tests as an example.

\section{C.1. Introduction}

The general logic used in the selection of the parameters and the design of the frame formats is explained for each case within the TCP/IP section. The same logic has been used in the other sections. Comments are used in these sections only if there is a protocol specific feature to be explained. Parameters and frame formats for additional protocols can be defined by the reader by using the same $\operatorname{logic}$.

C.2. TCP/IP Information

The following section deals with the TCP/IP protocol suite.

C.2.1 Frame Type.

An application level datagram echo request is used for the test data frame in the protocols that support such a function. A datagram protocol is used to minimize the chance that a router might expect a specific session initialization sequence, as might be the case for a reliable stream protocol. A specific defined protocol is used because some routers verify the protocol field and refuse to forward unknown protocols.

For TCP/IP a UDP Echo Request is used.

\section{C.2.2 Protocol Addresses}

Two sets of addresses must be defined: first the addresses assigned to the router ports, and second the address that are to be used in the frames themselves and in the routing updates.

The network addresses 192.18.0.0 through 192.19.255.255 are have been assigned to the BMWG by the IANA for this purpose. This 
assignment was made to minimize the chance of conflict in case a testing device were to be accidentally connected to part of the Internet. The specific use of the addresses is detailed below.

\section{C.2.2.1 Router port protocol addresses}

Half of the ports on a multi-port router are referred to as "input" ports and the other half as "output" ports even though some of the tests use all ports both as input and output. A contiguous series of IP Class C network addresses from 198.18.1.0 to 198.18.64.0 have been assigned for use on the "input" ports. A second series from 198.19.1.0 to 198.19.64.0 have been assigned for use on the "output" ports. In all cases the router port is node 1 on the appropriate network. For example, a two port DUT would have an IP address of 198.18.1.1 on one port and 198.19.1.1 on the other port.

Some of the tests described in the methodology memo make use of an SNMP management connection to the DUT. The management access address for the DUT is assumed to be the first of the "input" ports (198.18.1.1).

\section{C.2.2.2 Frame addresses}

Some of the described tests assume adjacent network routing (the reboot time test for example). The IP address used in the test frame is that of node 2 on the appropriate Class $\mathrm{C}$ network. (198.19.1.2 for example)

If the test involves non-adjacent network routing the phantom routers are located at node 10 of each of the appropriate Class $\mathrm{C}$ networks. A series of Class $\mathrm{C}$ network addresses from 198.18.65.0 to 198.18.254.0 has been assigned for use as the networks accessible through the phantom routers on the "input" side of DUT. The series of Class C networks from 198.19.65.0 to 198.19.254.0 have been assigned to be used as the networks visible through the phantom routers on the "output" side of the DUT.

\section{C.2.3 Routing Update Frequency}

The update interval for each routing protocol is may have to be determined by the specifications of the individual protocol. For IP RIP, Cisco IGRP and for OSPF a routing update frame or frames should precede each stream of test frames by 5 seconds. This frequency is sufficient for trial durations of up to 60 seconds. Routing updates must be mixed with the stream of test frames if longer trial periods are selected. The frequency of updates should be taken from the following table. 


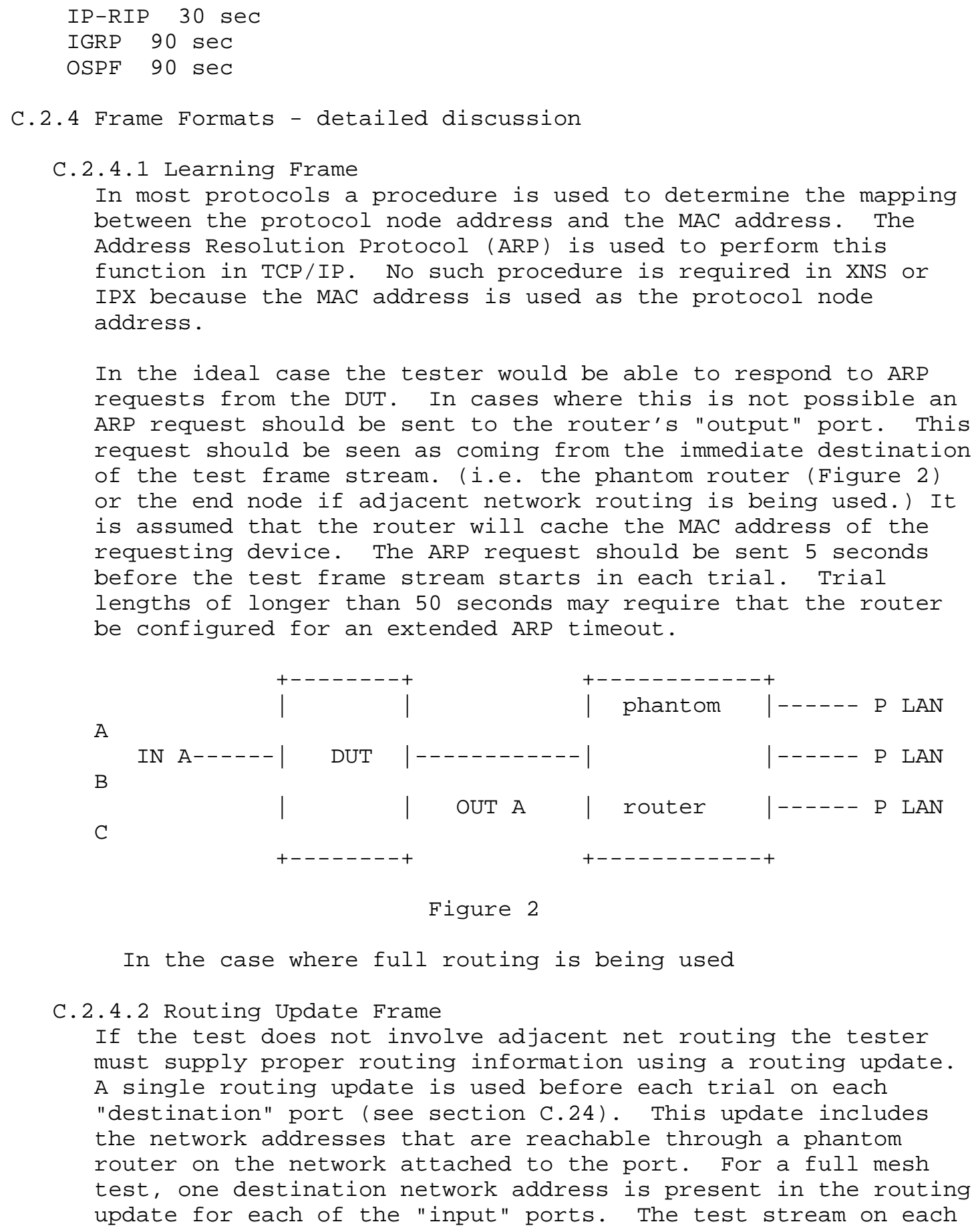

A

Figure 2

In the case where full routing is being used

\section{C.2.4.2 Routing Update Frame}

If the test does not involve adjacent net routing the tester must supply proper routing information using a routing update. A single routing update is used before each trial on each "destination" port (see section C.24). This update includes the network addresses that are reachable through a phantom router on the network attached to the port. For a full mesh test, one destination network address is present in the routing update for each of the "input" ports. The test stream on each 
"input" port consists of a repeating sequence of frames, one to each of the "output" ports.

C.2.4.3 Management Query Frame

The management overhead test uses SNMP to query a set of variables that should be present in all DUTs that support SNMP. The variables for a single interface only are read by an NMS at the appropriate intervals. The list of variables to retrieve follow:

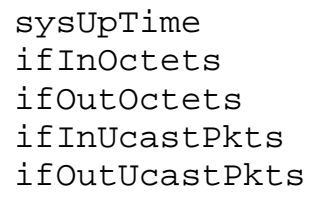


32

38
FF $\quad F F \quad F F \quad F F \quad F F \quad F F$

$X X \quad X X \quad X X$ requesting $\mathrm{DUT}^{\prime} \mathrm{S}$ MAC address DUT'S IP address
C.2.6.2 Routing Update Frame

\begin{tabular}{|c|c|c|}
\hline $\begin{array}{l}--D A T \\
\text { offset }\end{array}$ & $\begin{array}{l}\text { TAGRA } \\
t \text { dat }\end{array}$ & \\
\hline 00 & $\mathrm{FF}$ & $\mathrm{FF}$ \\
\hline 06 & $x x$ & $x x$ \\
\hline 12 & 08 & 00 \\
\hline$--I P$ & HEAD & \\
\hline 14 & 45 & \\
\hline byte un & nits) & \\
\hline 15 & 00 & \\
\hline 16 & 00 & $\mathrm{EE}$ \\
\hline 18 & 00 & 00 \\
\hline 20 & 40 & 00 \\
\hline fragmen & $n t)$ & \\
\hline
\end{tabular}

\begin{tabular}{|c|c|c|c|}
\hline 22 & $O A$ & & \\
\hline 23 & 11 & & \\
\hline 24 & C4 & $8 D$ & \\
\hline 26 & $x x$ & $x x$ & $x x$ \\
\hline 30 & $x x$ & $x x$ & $x x$ \\
\hline 33 & $\mathrm{FF}$ & & \\
\hline
\end{tabular}

-- UDP HEADER

$\begin{array}{lll}34 & 02 & 08\end{array}$

$\begin{array}{lll}36 & 02 & 08\end{array}$

$38 \quad 00 \mathrm{DA}$

$40 \quad 00 \quad 00$

- RIP packet

$42 \quad 02$

$43 \quad 01$

$44 \quad 00 \quad 00$

-- net 1

$46 \quad 00 \quad 02$

$48 \quad 00 \quad 00$

$50 \quad \mathrm{xx} x \mathrm{xx}$

$53 \quad 00$

$\begin{array}{lllll}54 & 00 & 00 & 00 & 00\end{array}$

$\begin{array}{lllll}58 & 00 & 00 & 00 & 00\end{array}$

$\begin{array}{lllll}62 & 00 & 00 & 00 & 07\end{array}$

-- net 2 description

dest MAC address is broadcast

source hardware address

type

IP version - 4, header length (4

service field

total length

ID

flags ( 3 bits) 4 (do not

fragment offset-0

TTL

protocol - 17 (UDP)

header checksum

source IP address

destination IP address

host part $=\mathrm{FF}$ for broadcast

source port $208=$ RIP

destination port $208=\mathrm{RIP}$

UDP message length

UDP checksum

command $=$ response

version $=1$

0

family $=$ IP

0

net 1 IP address

net not node

0

0

metric 7 


\begin{tabular}{|c|c|c|c|c|c|}
\hline 66 & 00 & 02 & & & family $=$ IP \\
\hline 68 & 00 & 00 & & & 0 \\
\hline 70 & $x x$ & $\mathrm{xx}$ & $x x$ & & net 2 IP address \\
\hline 73 & 00 & & & & net not node \\
\hline 74 & 00 & 00 & 00 & 00 & 0 \\
\hline 78 & 00 & 00 & & 00 & 0 \\
\hline 82 & 00 & 00 & 00 & 07 & metric 7 \\
\hline-- net & 3 & & & & \\
\hline 86 & 00 & 02 & & & family $=$ IP \\
\hline 88 & 00 & 00 & & & 0 \\
\hline 90 & $\mathrm{xx}$ & $\mathrm{xx}$ & $x x$ & & net 3 IP address \\
\hline 93 & 00 & & & & net not node \\
\hline 94 & 00 & 00 & 00 & 00 & 0 \\
\hline 98 & 00 & 00 & 00 & 00 & 0 \\
\hline 102 & 00 & 00 & 00 & 07 & metric 7 \\
\hline-- net & 4 & & & & \\
\hline 106 & 00 & 02 & & & family $=I P$ \\
\hline 108 & 00 & 00 & & & 0 \\
\hline 110 & $x x$ & $x x$ & $x x$ & & net 4 IP address \\
\hline 113 & 00 & & & & net not node \\
\hline 114 & 00 & 00 & 00 & 00 & 0 \\
\hline 118 & 00 & 00 & 00 & 00 & 0 \\
\hline 122 & 00 & 00 & 00 & 07 & metric 7 \\
\hline-- net & 5 & & & & \\
\hline 126 & 00 & 02 & & & family $=I P$ \\
\hline 128 & 00 & 00 & & & 0 \\
\hline 130 & 00 & & & & net 5 IP address \\
\hline 133 & 00 & & & & net not node \\
\hline 134 & 00 & 00 & 00 & 00 & 0 \\
\hline 138 & 00 & 00 & 00 & 00 & 0 \\
\hline 142 & 00 & 00 & 00 & 07 & metric 7 \\
\hline-- net & 6 & & & & \\
\hline 146 & 00 & 02 & & & family $=$ IP \\
\hline 148 & 00 & 00 & & & \\
\hline 150 & $x x$ & $x x$ & $x x$ & & net 6 IP address \\
\hline 153 & 00 & & & & net not node \\
\hline 154 & 00 & 00 & 00 & 00 & 0 \\
\hline 158 & 00 & 00 & 00 & 00 & 0 \\
\hline 162 & 00 & 00 & 00 & 07 & metric 7 \\
\hline
\end{tabular}




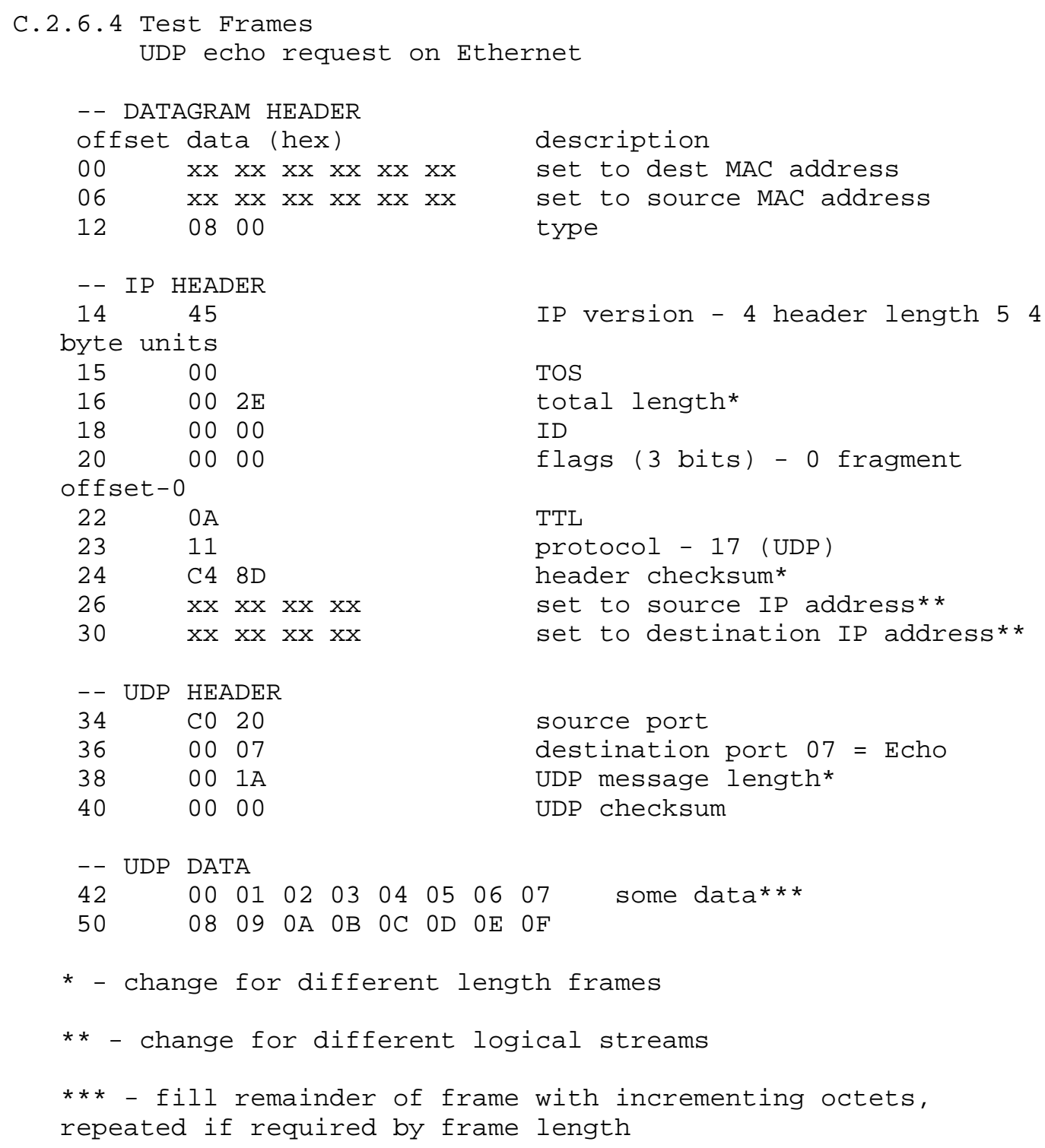




Values to be used in Total Length and UDP message length fields:
$\begin{array}{ccc}\text { frame size } & \text { total length } & \text { UDP message length } \\ 64 & 002 \mathrm{E} & 001 \mathrm{~A} \\ 128 & 00 \mathrm{E} & 005 \mathrm{~A} \\ 256 & 00 \mathrm{EE} & 009 \mathrm{~A} \\ 512 & 01 \mathrm{EE} & 019 \mathrm{~A} \\ 768 & 02 \mathrm{EE} & 029 \mathrm{~A} \\ 1024 & 03 \mathrm{EE} & 039 \mathrm{~A} \\ 1280 & 04 \mathrm{EE} & 049 \mathrm{~A} \\ 1518 & 05 \mathrm{DC} & 05 \mathrm{C} 8\end{array}$

